\title{
Evolution thermique de la structure magnétique dans $\mathrm{Tb}_{3} \mathrm{Fe}_{5} \mathrm{O}_{12}$
}

\author{
M. Lahoubi, G. Fillion* et F. Tchéou**
}

Laboratoire de Magnétisme, Institut de Physique, Université de Annaba, BP. 12, El-Hadjar, 23000 Annaba, Algérie

* Laboratoire de Magnétisme Louis Néel, CNRS-UJF, 25 avenue des Martyrs, B.P 166, 38042 Grenoble cedex 9, France

** GETA-IMAG, UJF 385, avenue de la Bibliothèque, Domaine Universitaire Saint-Martin-d'Hères, BP. 53X, 38041 Grenoble cedex, France

\begin{abstract}
Résumé. L'étude de l'évolution thermique de la structure magnétique du ferrite grenat de terbium $\left(\mathrm{Tb}_{3} \mathrm{Fe}_{5} \mathrm{O}_{12}\right)$ a été réalisée par diffraction neutronique entre $4.2 \mathrm{~K}$ et $614 \mathrm{~K}$. Elle a permis de mettre en évidence en dessous de $130 \mathrm{~K}$, l'apparition de la structure non colinéaire "en double parapluie" qui existe à $4.2 \mathrm{~K}$. Son évolution thermique vers une structure colinéaire de Néel au dessus de $130 \mathrm{~K}$ est continue. L'application des modèles théoriques de l'analyse de représentation du groupe d'espace rhomboédrique $(\mathrm{R} \overline{\mathrm{c}} \mathrm{c})$ avec $\mathrm{k}=[000]$ a montré qu'à toute température entre $4.2 \mathrm{~K}$ et la température d'ordre $T_{\mathrm{N}}(560 \mathrm{~K})$ la structure magnétique de $\mathrm{TbIG}$ appartient à la seule représentation irréductible à une dimension $\Gamma_{2 \mathrm{~g}}\left(\mathrm{~A}_{2 \mathrm{~g}}\right)$ pour laquelle les caractères des éléments générateurs $3, \overrightarrow{1}$, c sont respectivement $+1,+1,-1:$ le groupe de Shubnikov correspondant est $\left(R \overrightarrow{3} c^{\prime}\right)$.
\end{abstract}

\section{ASPECT EXPERIMENTAL}

Des expériences de diffraction des neutrons ont été réalisées sur le détecteur "P.S.D" (Position - Sensitive -Detector) installé au réacteur "Siloé" du Centre d'Etude Nucléaire de Grenoble. Les mesures ont été effectuées sur un échantillon de TbIG polycristallin en utilisant un faisceau de neutrons monochromatique ayant $2.49 \AA$ pour longueur d'onde. Dans un premier temps, nous avons utilisé un appareillage cryogénique qui nous a permis d'enregistrer dix diagrammes dans la zone de température : $4.2 \mathrm{~K}-300 \mathrm{~K}$. De plus, des précautions ont été prises afin d'éviter la contamination des diagrammes par des raies parasites dues aux réflexions sur les parois du cryostat. Dans un deuxième temps, l'utilisation d'un four nous a permis d'enregistrer des diagrammes de neutrons à des températures comprises entre $300 \mathrm{~K}$ et $614 \mathrm{~K}$. Des précautions ont été également prises afin de réduire la contamination des spectres obtenus par des raies associées à la réflexion de l'harmonique $\lambda / 2$ et qui sont non négligeables aux faibles angles.

\section{RESULTATS ET DISCUSSION}

Nous avons obtenu à $4.2 \mathrm{~K}$ [1] de nouveaux paramètres de la structure magnétique de TbIG. Nous avons montré que cet arrangement "en double parapluie" a conduit à un accord très satisfaisant en appliquant le modèle de structure magnétique rhomboédrique "en parapluie $(e),\left(e^{\prime}\right)$ " de la représentation irréductible à une dimension $\Gamma_{2 g}\left(A_{2 g}\right)$ du groupe d'espace $R \overline{3} c-$ $\left(\mathrm{D}_{3 d}^{6}\right)$ avec $\mathbf{k}=[000]$ [2]. Nous avons donc tenté d'interpréter les résultats expérimentaux obtenus aussi bien à basse qu'à haute température en utilisant ce modèle de structure magnétique.

Nous avons reporté sur la figure 1 , la variation thermique des intensités magnétiques calculée et observée de la raie de surstructure $(310)^{*}$. On constate que l'accord entre $\left(\mathrm{I}_{\mathrm{M}}\right)_{c a l}$ et $\left(\mathrm{I}_{\mathrm{M}}\right)_{\text {obs }}$ est très satisfaisant entre $4.2 \mathrm{~K}$ et $130 \mathrm{~K}$. De plus, ces intensités magnétiques présentent une variation rapide vers $50 \mathrm{~K}$. Ce résultat est à comparer à celui obtenu par Sayetat [3] qui a mis en évidence une déformation rhomboédrique qui apparât sans ambiguiité à partir de $100 \mathrm{~K}$ avec plus de netteté au voisinage de $45 \mathrm{~K}$. L'intensité magnétique $\mathrm{I}_{M}$ d'une raie de structure peut se déduire aisément en retranchant la contribution nucléaire $\mathrm{I}_{\mathrm{N}}$ (qui varie peu avec la température) à l'intensité totale $\mathrm{I}$. Cette définition permet la détermination de $\mathrm{I}_{\mathrm{M}}$ avec une précision relativement bonne pour des raies à forte contribution magnétique telle que (211). La variation thermique des intensités magnétiques calculée et observée de cette réflexion a été également reportée sur cette figure . La comparaison montre qu'elles sont en bon accord entre $4.2 \mathrm{~K}$ et $453 \mathrm{~K}$. Cette raie (211) qui apparaît à la température de Néel $\mathrm{T}_{\mathrm{N}}$ est responsable de l'ordre ferrimagnétique colinéaire. Elle augmente lorsque la température diminue et présente une variation rapide au voisinage de $130 \mathrm{~K}$. 

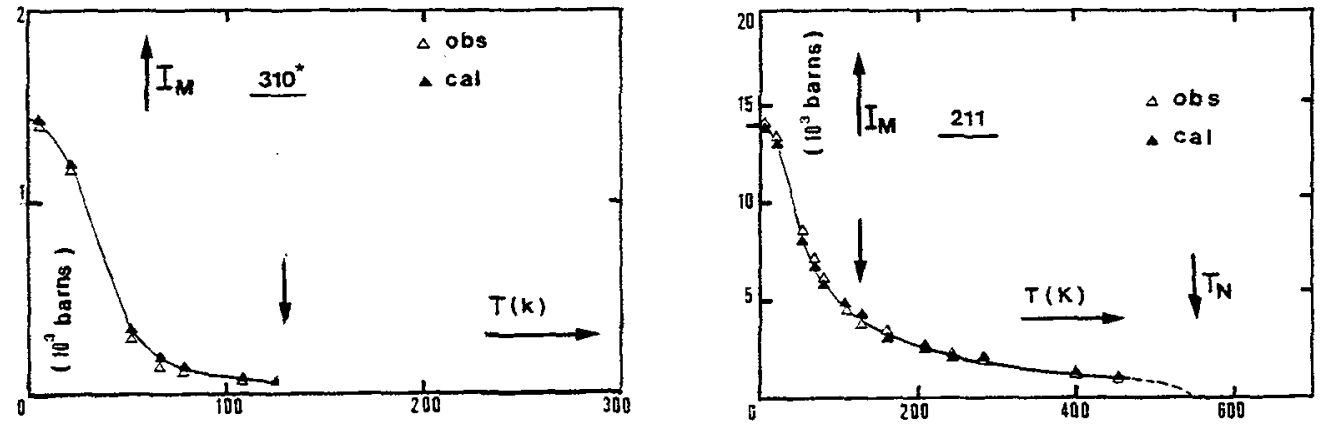

Figure 1 : Variation thermique des intensités magnétiques calculées et observées des raies (310)* et (211).
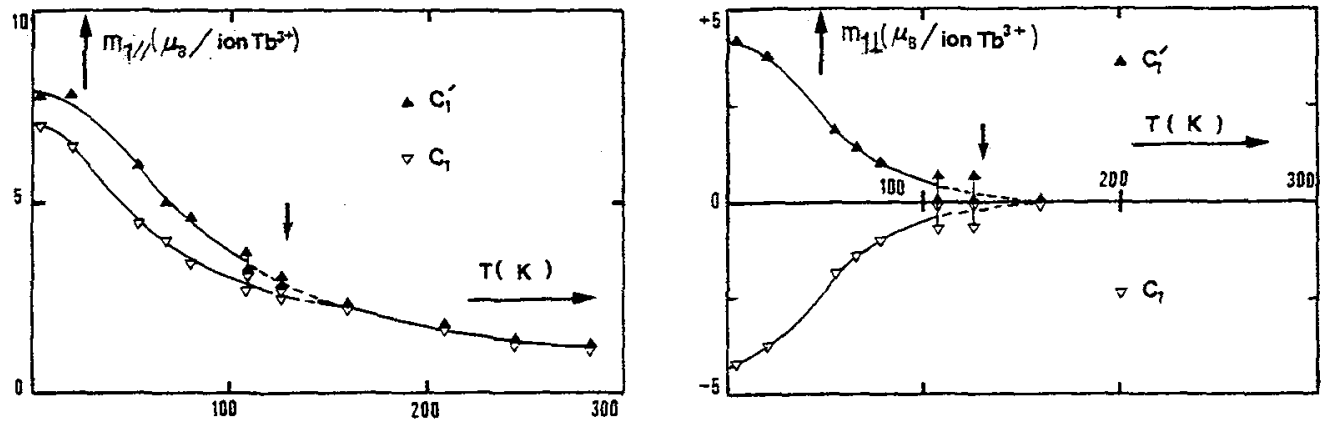

Figure 2 : Variation thermique des composantes parallèles $\left(m_{1 / /}, m_{l}^{\prime}\right)$ et perpendiculaires $\left(m_{1 \perp}, m_{1}^{\prime}\right)$ des moments magnétiques.

La variation thermique des composantes parallèles $\left(\mathbf{m}_{1 / j}, \mathbf{m}_{1}^{\prime} /\right)$ et perpendiculaires $\left(\mathbf{m}_{1 \perp}, \mathbf{m}_{1}^{\prime}\right)$ des moments magnétiques $m_{1}\left(C_{1}\right)$ et $m_{1}{ }^{\prime}\left(C_{1}{ }^{\prime}\right)$ est reportée sur la figure 2 . Au dessus de $130 K$, les composantes $m_{1 \perp}$ et $m_{1}{ }^{\prime}$ sont nulles et les moments magnétiques de terbium alignés suivant [111] forment un seul sous réseau magnétique. Au dessous de 130K, conjointement à l'apparition des deux composantes perpendiculaires et opposées, nous constatons que les composantes $\mathbf{m}_{1 / /}$ et $\mathrm{m}_{1 / /}^{\prime}$ qui étaient initialement égales augmentent progressivement en se différenciant lorsque la température diminue jusqu'à $4.2 \mathrm{~K}$ où nous avons : (6e) $\mathrm{C}_{1} ; \mathrm{m}_{1}: \mathrm{m}_{1 / /}=+7.03 \mu_{\mathrm{B}}$ et $\mathrm{m}_{1 \perp}=-4.19 \mu_{\mathrm{B}},(6 \mathrm{e}) \mathrm{C}_{1}{ }^{\prime} ; \mathrm{m}_{1}^{\prime}: \mathrm{m}_{1 / 1}^{\prime}=+7.85 \mu_{\mathrm{B}}$ et $\mathrm{m}_{1}{ }^{\prime}=+4.19 \mu_{\mathrm{B}}$.

Cette température caractéristique $(T=130 \pm 30 \mathrm{~K})$ à laquelle l'ouverture spontanée des moments de terbium semble se produire possède seulement une signification indicative car le passage du modèle colinéaire de Néel au modèle non colinéaire "en double parapluie" ne constitue pas une transition de phase. Dans toute la gamme de température $\left(4.2 \mathrm{~K}-\mathrm{T}_{\mathrm{N}}\right)$, la symétrie magnétique ne change pas avec la température, le groupe magnétique $\mathrm{R} \overline{3} \mathrm{c}^{\prime}$ demeure invariant et la seule représentation irréductible à une dimension $\Gamma_{2 g}\left(A_{2 g}\right)$ permet d'expliquer la déformation rhomboédrique qui peut exister dès ' $T_{N}$.

\section{Remerciements}

Nous remercions M. Guillot (LCMI Grenoble) de nous avoir fourni l'échantillon.

\section{Références}

[1] Lahoubi M., Guillot M., Marchand A., Tchéou F. and Roudaut E., L.E.E.E. Trans. Magn.Mag 20 (1984) 1518-1520.

[2] Lahoubi M., Structures magnétiques non colinéaires et transitions de phases dans les grenats ferrimagnétiques de Terbium et d'Yttrium (Thèse de Doctorat d'Etat, Université Joseph Fourier, 1986) pp. 75-127.

[3] Sayetat F., J.M.MM. 58 (1986) 334-346. 